\title{
Rapid Variations in the Seyfert 1 Galaxy Mrk 474
}

\author{
R. R. Valiullin \\ Fesenkov Astrophysical Institute, Kamenskoye Plato, Almaty, 480020, \\ Kazakhstan. Electronic mail: rashit@afi.south-capital.kz
}

\begin{abstract}
Spectra of Mrk 474 have been obtained in order to search for short-term (less than one day) variability in the broad $\mathrm{H} \beta$ and $\mathrm{H} \alpha$ profiles. Observations were carried out using a slit spectrograph and a three-cascade image tube attached to the $70-\mathrm{cm}$ reflector. The processing of the series of spectrograms, obtained on 1999 April 25, showed a rapid (less than 20 minutes) and powerful increase of the flux in the region of $[\mathrm{O}$ III $] \lambda 5007$. The increase of the flux near maximum $(5060 \AA \pm 10 \AA)$ appeared to be equal approximately to $300 \%$. The value similar to the FWHM for the long-wavelength excess of the flux was equal to $170 \AA \pm$ $20 \AA$. The dying-out of the long-wavelength excess lasted approximately two hours.
\end{abstract}

\section{Analysis and Results}

1. The third spectrum of the series if compared with the first and the second ones has a significant excess of the flux above the 'average' spectrum in the region of the $[\mathrm{O}$ III $] \lambda 5007$ emission line. The maximum of a long-wave flux excess corresponds to the $5060 \AA \pm 10 \AA$. The increase of the flux near the maximum of the long-wavelength excess $(5060 \AA$ ) appeared to be approximately equal to $300 \%$. The value similar to the FWHM for the long-wavelength excess appeared to be equal to $170 \AA \pm 20 \AA$. The dying-out of the long-wavelength flux excess lasted approximately two hours with a variable velocity: firstly, during a transition from the third spectrum of the series to the fourth one, it goes quickly, and then-slower. The position of the center of symmetry of the long-wavelength flux excess during the transition from the third spectrum of the series to the seventh one gradually shifted towards the center of the $\mathrm{H} \beta$ emission line.

2. During the transition from the fifth spectrum of the series to the sixth, a noticeable excess of the flux appeared in two regions. The center of symmetry of the first short-wavelength flux excess corresponds to $4750 \AA \pm 10 \AA$, and the increase of the flux in the first short-wavelength excess near $4750 \AA$ appeared to be equal approximately to $200 \%$. The value similar to the FWHM for the first short-wavelength excess appeared to be equal to $100 \AA \pm 20 \AA$. The center of symmetry of the second excess is obviously less than the short-wavelength edge of our observed wavelength region i.e. it is $<4540 \AA$. Therefore it is difficult to estimate precisely the increase of the flux in the second excess, but most likely it is not less than $200 \%$ at its maximum. 


\section{Discussion}

During observations of variations in the spectrum of Mrk 474 the fact was registered that the short-wavelength excess of the flux appeared 77 minutes later than the long-wavelength excess. In addition, the period of motion of the center of symmetry of the flux excess near the $\mathrm{H} \beta$ emission line is also equal to $77 \mathrm{~min}-$ utes. Thus one can conclude that these two events are interrelated. The reason for the origin of one long-wavelength and two short-wavelength excesses and a flux excess near the $\mathrm{H} \beta$ emission line may be a reverberation of the hydrogen clouds from a sudden flare in the UV continuum (see e.g., Blandford \& McKee 1982; Peterson 1997). This assumption may explain also the shift of the center of symmetry of the long-wavelength flux excess towards shorter wavelength. With propagation of a flare from the central source of continuum, the flare process would gradually involve the more distant clouds of hydrogen, the velocities of which are decreasing with distance from the central source. Specifically, while the intensity of radiation on rapidly moving hydrogen clouds located close to the source of continuum decreases, the intensity of radiation on the more remote, and therefore, slower hydrogen clouds increases. The effects mentioned above will result in a redistribution of the spectral flux and a shift of the center of symmetry of the radiation excess towards a wavelength corresponding to zero velocity.

If our assumption is correct, a model can be considered, where three large hydrogen clouds revolve along elliptic orbits around the source of continuum. For the observer two of them are behind the source of continuum and are moving towards the observer with radial velocities $-7480 \pm 600 \mathrm{~km} \mathrm{~s}^{-1}$ and $-19814 \pm$ ? $\mathrm{km} \mathrm{s}^{-1}$ respectively, and one cloud is in front of the central source and moves away from the observer with a radial velocity $12250 \pm 600 \mathrm{~km} \mathrm{~s}^{-1}$. These assigned values are related only to that part of the hydrogen which produces radiation near the maximum of the flux excess. There is a dispersion of velocities inside each hydrogen cloud. The range of velocities may reach several thousand $k m s^{-1}$ and the more distant fragments would have the smaller velocities. The presence of the slower parts of the clouds is necessary for explaining the shift of the center of symmetry of the radiation excess towards the wavelength of the relative zero velocity of hydrogen.

\section{References}

Blandford, R. D., \& McKee, C. F. 1982, ApJ, 255, 419

Denissyuk, E. K. 1974, Astron. Zh., No 809, 1

Peterson, B.M. 1997, An introduction to Active Galactic Nuclei (Cambridge: Cambridge Univ. Press), 82 